Nouvelles perspectives en sciences sociales

Revue internationale de systémique complexe et d'études relationnelles

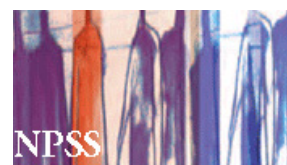

\title{
Recherche interdisciplinaire et réflexion sur l'interdisciplinarité
}

\section{Simon Laflamme}

Volume 7, numéro 1, octobre 2011

Sur le thème de l'interdisciplinarité

URI : https://id.erudit.org/iderudit/1007081ar

DOI : https://doi.org/10.7202/1007081ar

Aller au sommaire du numéro

Éditeur(s)

Prise de parole

ISSN

1712-8307 (imprimé)

1918-7475 (numérique)

Découvrir la revue

Citer cet article

Laflamme, S. (2011). Recherche interdisciplinaire et réflexion sur l'interdisciplinarité. Nouvelles perspectives en sciences sociales, 7(1), 49-64. https://doi.org/10.7202/1007081ar
Résumé de l'article

L'interdisciplinarité constitue une pratique scientifique usuelle et féconde. Il y a d'ailleurs de nombreuses théories interdisciplinaires. Mais il n'y a pas de théorie de l'interdisciplinarité. Les études sur l'interdisciplinarité parlent d'une approche appelée à sauver le monde, dichotomisent monodisciplinarité et interdisciplinarité, fabriquent des typologies du travail interdisciplinaire, mais elles ne livrent pas de théories de l'interdisciplinarité. En fait, le projet d'une telle théorie est absurde parce que s'il peut y avoir des théories scientifiques, il ne peut y avoir de théorie de la science. C'est ce que soutient cet article. 


\title{
Recherche interdisciplinaire et réflexion sur l'interdisciplinarité
}

\author{
Simon LafLAmme \\ Université Laurentienne, Sudbury
}

\section{Une question}

ans leur appel de textes, Rachid Bagaoui et Alain Beaulieu invitent les collaborateurs à répondre à la question suivante : Y a-t-il une théorie de l'interdisciplinarité en sciences humaines? Nous répondons à cette question par la négative. Avant de livrer cette réponse, nous commençons par rappeler que la recherche interdisciplinaire fait clairement partie du paysage des sciences humaines, puis nous décrivons ce qui nous semble constituer l'essentiel des réflexions sur l'interdisciplinarité.

\section{L'interdisciplinarité comme constituante des sciences humaines}

La question de l'interdisciplinarité, en sciences humaines comme dans tout le champ scientifique, en fait, - n'est certainement pas celle de la pertinence ou de la légitimité. Pour en faire la démonstration, si besoin est encore, on peut rappeler que bon nombre des grands théoriciens représentent des carrefours où se rencontrent diverses disciplines. Chez Karl Marx se croisent la philosophie, l'histoire et l'économie politique. George H. Mead 
conjugue la psychologie, la sociologie et la philosophie. Chez Claude Lévi-Strauss se marient la linguistique et l'anthropologie. Michel Foucault jongle avec l'histoire, la psychologie et la philosophie. Chez Niklas Luhmann s'entrechoquent sociologie, sciences de la communication et philosophie, tout comme chez Jürgen Habermas, par ailleurs. Et chacune de ces œuvres a des échos dans toutes les sciences humaines. On peut aussi énumérer les nombreux champs de connaissance qui n'ont d'existence que parce qu'ils réunissent plusieurs disciplines, comme les sciences de la communication, les études sur la femme, les sciences cognitives, les sciences de l'environnement, le développement humain, les études régionales, les relations industrielles, l'aménagement territorial, l'orthophonie, les sciences de la santé ${ }^{1}$. On peut encore mentionner d'innombrables intersections qu'a créées l'histoire des sciences : psychologie sociale, sociolinguistique, sociohistoire, géographie politique, anthropologie physique, psychoéducation... On peut, de surcroît, signaler les multiples centres de recherche dans les universités et hors d'elles dans lesquels se côtoient les chercheurs de divers horizons et dans lesquels abondent les produits scientifiques de qualité, développés à la jonction de plusieurs disciplines.

L'interdisciplinarité est un fait, une manifestation notoire de l'histoire des sciences. Ses critiques, d'ailleurs, ne la nient jamais. Ils la craignent. Ils craignent, par exemple, qu'un doctorat interdisciplinaire ne procure pas au diplômé de poste dans une université. Il est vrai que la tradition universitaire, par sa structuration, freine le travail interdisciplinaire. Les historiens embauchent des historiens, les psychologues, des psychologues, les géographes, des géographes, et, pour cette raison, l'université se reproduit largement dans la monodisciplinarité. Mais dans sa quête de vérité, l'université est aussi tournée vers l'interdisciplinarité. C'est la raison pour laquelle elle crée de plus en plus de programmes dans lesquels peut s'exprimer cette aspiration; on ne

C'est en se penchant sur ces domaines d'études que certains en viennent à imaginer une recherche méta, ou post, ou in, ou para, ou $a$, ou extradisciplinaire. 
peut pas comprendre l'environnement, ou la femme, ou les premières nations, ou les relations industrielles dans un cadre unidisciplinaire. C'est aussi pourquoi elle favorise les liens entre les chercheurs de diverses disciplines, car s'il est facile de procéder à des observations dans un cadre monodisciplinaire, il est plus difficile de théoriser dans ce strict milieu scientifique : la psychologie peut bien associer l'estime de soi et le quotient intellectuel, mais elle ne rendra pas aisément compte de cette corrélation positive sans emprunter à la sociologie. Et puis rares sont les objets dont les caractéristiques ne puissent être appréhendées que par une discipline, et moins l'objet est modélisé, plus il appelle l'interdisciplinarité. La ville, la honte, le chômage, la déviance, l'ambition sont de ces objets, peu modélisés parce qu'ils appartiennent au champ de la vie en société davantage qu'à la science, qui sont d'autant mieux compris que l'analyse n'est pas monodisciplinaire. L'université est donc moins centrée sur l'unidisciplinarité qu'on aime à le dire. Elle est certes tournée vers la spécialisation, mais toute spécialisation n'est pas unidisciplinaire. Nous pensons avec Peter Weingart que l'interdisciplinarité est aussi bienvenue qu'elle est malvenue dans les universités; mais nous pensons aussi avec lui que monodisciplinarité et interdisciplinarité évoluent de pair, l'une et l'autre s'interpellant, les conclusions monodisciplinaires réclamant des recompositions, les analyses interdisciplinaires sollicitant des déconstructions, sans que cela n'affecte l'autonomie relative de chacune d'elles ${ }^{2}$. Et dans chacun de ces milieux, la science produit des travaux impressionnants. Les œuvres sont aussi belles dans les disciplines respectives qu'à leurs intersections.

\section{Les réflexions sur l'interdisciplinarité}

Le travail interdisciplinaire est donc évident, et il est inscrit dans le champ scientifique, il fait corps avec lui. Bon nombre de penseurs ont constaté cette évidence; bon nombre, inquiétés par

Peter Weingart, «Interdisciplinarity: The Paradoxical Discourse », dans Peter Weingart et Nico Stehr (dir.), Practising Interdisciplinarity, Toronto, Buffalo, London, University of Toronto Press, 2000, p. 25-41. 
l'ampleur de l'espace occupé par le travail monodisciplinaire dans le champ scientifique en ont appelé au travail interdisciplinaire. À nos yeux, ces réflexions se sont principalement engagées dans trois voies.

3.1. L'interdisciplinarité pour sauver le monde

Dans la première voie, des critiques s'en prennent à la spécialisation. Il s'agit, par exemple, d'un Giambattista Vico qui, dès 1725 , ne veut pas que la tendance à la subdivision des savoirs empêche que les individus acquièrent une connaissance générale ${ }^{3}$. Il s'agit aussi, en 1886, d'un Friedrich Nietzsche qui exprime son mépris contre les " hommes de science ", une "variété roturière de l'humanité ", leur reprochant d'être des " ouvriers de la philosophie ", et donc de ne pas être de "véritables philosophes", c'est-à-dire ceux pour lesquels la " "connaissance" est création ${ }^{4}$ ". Il s'agit encore d'un José Ortega y Gasset qui, en 1930, dénonce " la barbarie du "spécialisme" » qui caractérise «l'homme scientifique ", cet homme " reclus dans l'étroitesse de son champ visuel ", « intellectuellement moyen " 5 .

Le mouvement vers la spécialisation des savoirs a vite inquiété. Des observateurs ont craint, au plan épistémologique, un éclatement des connaissances, et, au plan humain, un défaut de culture générale.

C'est Edgar Morin qui a sans doute le mieux exprimé cette préoccupation. Il l'a fait, d'abord, en plaidant souvent pour une culture intégrée des acteurs sociaux ${ }^{6}$. Il l'a fait, ensuite, en mettant en lumière le cartésianisme sur lequel repose largement l'évolution de la science, évolution qui s'alimente aux principes de

3 Giambattista Vico, La science nouvelle, Paris, Gallimard, coll. « Tel », [1725] 1993.

4 Friedrich Nietzsche, « Nous, les savants », dans Par-delà le bien et le mal, Paris, Union générale d'éditions, coll. "10/18 ", [1725] 1970, p. 133-152.

5 José Ortega y Gasset, "La barbarie du "spécialisme" ", dans La révolte des masses, Paris, Gallimard, coll. «Idées », [1930] 1961, p. 158-159.

6 Edgar Morin, Science et conscience, Paris, Seuil, coll. "Points Essais ", 1990; La tête bien faite, Paris, Seuil, 1999; La méthode, tome 5, L'humanité de l'humanité, Paris, Seuil, 2001; La méthode, tome 6, L'éthique, Paris, Seuil, 2006. 
séparabilité et de réduction et qui se nourrit de la logique aristotélicienne. Il l'a fait, troisièmement, en signalant ce qui échappait à cette perspective : le désordre, la non-séparabilité, l'incertitude logique. Il l'a fait, quatrièmement, en reconnaissant l'importance du cartésianisme, mais en proposant une autre philosophie, qu'il dira complexe, dans laquelle 1) les logiques récursive et linéaire peuvent coexister, 2) les éléments contraires ne constituent pas forcément la négation les uns des autres, 3) les parties sont réintégrées dans le tout. Cette volonté de maintenir le lien entre les éléments se révèle chez lui de trois façons : à travers un projet hologrammatique où les parties sont dans le tout aussi bien que le tout est dans les parties; dans un emprunt à la systémique qui oblige à comprendre les éléments les uns par rapport aux autres et à interpréter le tout comme processus (auto-éco-ré-) organisateur; par une valorisation de l'interdisciplinarité

La critique de la spécialisation a atteint son point culminant en 1994 quand a été adoptée la Charte de la transdisciplinarité au "Premier congrès mondial de la transdisciplinarité ", le comité de rédaction ayant été formé de Lima de Freitas, Edgar Morin et Basarab Nicolescu. Pour en bien comprendre l'esprit, on peut en lire le préambule :

Considérant que la prolifération actuelle des disciplines académiques et non académiques conduit à une croissance exponentielle du savoir, ce qui rend impossible tout regard global de l'être humain,

Considérant que seule une intelligence qui rend compte de la dimension planétaire des conflits actuels pourra faire face à la complexité de notre monde et au défi contemporain d'autodestruction matérielle et spirituelle de notre espèce,

Considérant que la vie est lourdement menacée par une technoscience triomphante, n'obéissant qu'à la logique effrayante de l'efficacité pour l'efficacité,

7 Edgar Morin, La méthode, tomes 1 à 4, Paris, Seuil, 1977-1991; "L'épistémologie de la complexité ", dans Edgar Morin et Jean-Louis Le Moigne, L'intelligence de la complexité, Paris, L'Harmattan, coll. "Cognition et formation ", 1999, p. 43-169; "La pensée complexe, une pensée qui se pense ", dans Edgar Morin et Jean-Louis Le Moigne, L'intelligence de la complexité, Paris, L'Harmattan, coll. "Cognition et formation ", 1999, p. 247-267. 
Considérant que la rupture contemporaine entre un savoir de plus en plus accumulatif et un être intérieur de plus en plus appauvri mène à une montée d'un nouvel obscurantisme, dont les conséquences sur le plan individuel et social sont incalculables,

Considérant que la croissance des savoirs, sans précédent dans l'histoire, accroît l'inégalité entre ceux qui les possèdent et ceux qui en sont dépourvus, engendrant ainsi des inégalités croissantes au sein des peuples et entre les nations sur notre planète,

Considérant en même temps que tous les défis énoncés ont leur contrepartie d'espérance et que la croissance extraordinaire des savoirs peut conduire, à long terme, à une mutation comparable au passage des hominiens à l'espèce humaine. ${ }^{8}$

\subsection{L'interdisciplinarité comme élément d'un dualisme}

Qu'ils posent l'uninidisciplinarité comme bonne ou mauvaise, pour bon nombre de penseurs, l'interdisciplinarité apparaît souvent comme son contraire. La monodisciplinarité est de l'ordre de la séparation, l'interdisciplinarité, de l'intégration'. À leurs yeux, monodisciplinarité coïncide avec raisonnement scientifique, analyse quantitative; interdisciplinarité, avec accueil du paradoxe, pensée qualitative ${ }^{10}$. Monodisciplinarité correspond à unité et universalité; interdisciplinarité, à pluralité et hétérogénéité ${ }^{11}$. Monodisciplinarité, dans cet esprit, renvoie à holisme et synthèse; interdisciplinarité, à interrogation et invention. La monodisciplinarité, ici entendue comme chacune des sciences de la physis, est objective et institutionnelle; la transdisciplinarité

8 Charte de la transdisciplinarité, http://basarab.nicolescu.perso.sfr.fr/ciret/ chartfr.htm, 1994, Premier congrès mondial de la transdisciplinarité, site consulté en décembre 2010. Le discours est semblable dans le livre de Basarab Nicolescu intitulé La Transdisciplinarité. Manifeste, Monaco, Éditions du Rocher, coll. "Transdisciplinarité ", 1996.

9 Edgard Morin, La méthode, op. cit.; Moti Nissani, "Fruits, Salads and Smoothies: A Working Definition of Interdisciplinarity ", Journal of Education Thought, vol. 29, $\mathrm{n}^{\circ}$ 2, août 1995, p. 121-128.

10 Michael Finkenthal, Interdisciplinarity: Toward the Definition of a Metadiscipline?, New York, Peter Lang, coll. "American University Studies ", Series V, Philosophy, vol. 187, 2001.

11 Julie Thompson Klein, Interdisciplinarity: History, Theory, and Practice, Détroit, Wayne State University, 1990. 
répond aux questions philosophiques, elle est subjective et non institutionnelle ${ }^{12}$.

Ce dualisme s'inscrit parfois dans une perspective évolutive. Des auteurs, en effet, font coïncider la transdisciplinarité et l'interdisciplinarité avec des étapes de l'histoire. Chez Michael Finkenthal, la spécialisation, ou la division du monde en disciplines, appartient à la modernité, à une vision du monde unitaire, universelle; la postmodernité a, pour corollaire, l'individualisme, une complexification du monde, et l'interdisciplinarité représente une possibilité de souder entre elles des parties ${ }^{13}$. Chez Julie Thompson Klein, il y a aussi cette vision historique où l'époque de l'universalité ou de l'unité est celle de la spécialisation et où l'ère de l'hétérogénéité est celle de l'interdisciplinarité ${ }^{14}$.

Pour certains interprètes, l'interdisciplinarité est la possibilité pour l'humain de fusionner les savoirs, de relier conscience et science, art et science. Cette vison est commune dans les études féministes où la quête d'interdisciplinarité a souvent pour corollaire un projet moral d'égalité des sexes, un refus d'appréhender la femme comme s'il s'agissait d'un objet d'étude, un désir d'éliminer la distance entre le mode analytique et le vécu subjectif $^{15}$. Elle est présente aussi chez Edgar Morin qui veut que science et conscience se compénètrent ${ }^{16}$. On l'aperçoit chez Michel Camus qui veut marier la culture des sciences " dures" à celle des sciences humaines, qui, selon lui, « tien[nen]t [leur]

12 Michel Camus, "Au-delà des deux cultures : la voie transdisciplinaire ", dans Groupe 21, Mémoire du XXI siècle, Monaco, Éditions du Rocher, 1999, p. 25-34.

13 Michael Finkenthal, op. cit.

14 Julie Thompson Klein, op. cit. On notera toutefois que cela n'empêche pas Klein de faire remonter l'interdisciplinarité ailleurs, dans le même livre, à l'époque encyclopédique, et même avant.

15 Alma Walker Vinyard, "Identification and Self-Definition: A Twenty-First Century Agenda for Interdisciplinary and International Women's Studies ", dans Kate Conway-Turner, Suzanne Cherrin, Jessica Schiffman, and Kathleen Doherty Turkel (dir.), Women's Studies in Transition. The Pursuit of Interdisciplinarity, Newark, University of Delaware Press / London, Associated University Press, 1998, p. 92-101.

16 Edgar Morin, Science et conscience, op. cit.; "L'épistémologie de la complexité ", op. cit.; "La pensée complexe, une pensée qui se pense ", op. cit. 
autorité de l'expérience intérieure ${ }^{17}$. On la trouve chez Julie Thompson Klein qui, dans les cultural studies, entrevoit le lieu de la relation entre les activités intellectuelles et artistiques ${ }^{18}$.

\subsection{L'interdisciplinarité comme type}

Un vaste pan des études sur l'interdisciplinarité est formé de typologies. Creutzer Mathurin ${ }^{19}$, dans un texte publié en 2002, en a recensé les plus importantes, dont la plupart sont issues du célèbre séminaire de l'Organisation de coopération et de développement économiques (OCDE) :

i. celle de Roger Bastide ${ }^{20}$, qui distingue l'interdisciplinaire, du transculturel et du multidisciplinaire;

i. celle de Heinz Heckhausen ${ }^{21}$, dans laquelle on trouve six catégories : interdisciplinarité hétérogène, pseudointerdisciplinarité, interdisciplinarité auxiliaire, interdisciplinarité composite, interdisciplinarité complémentaire, interdisciplinarité unificatrice;

i. celle de Marcel H. Boisot ${ }^{22}$, dont les trois termes sont interdisciplinarité linéaire, interdisciplinarité structurale et interdisciplinarité restrictive;

ii. celle d'Erich Jantsch ${ }^{23}$, composée de trois ensembles, dont le premier est l'interdisciplinarité croisée (ou

17 Michel Camus, "Au-delà des deux cultures : la voie transdisciplinaire ", op. cit.

Julie Thompson Klein, Interdisciplinarity, op. cit.

19 Creutzer Mathurin, "Aspects de l'interdisciplinarité : essai de reconstitution d'un débat ", dans Lucie Gélineau et Carole Mailloux (dir.), Linterdisciplinarité et la recherche sociale appliquée. Réflexions sur des expériences en cours, Université de Montréal, Université Laval et Chaire d'étude Claire-Bonenfant sur la condition des femmes, octobre 2002, p. 7-39, http:/www.fas.umontreal.ca/ sha/documents/interdisciplinarite.pdf, site consulté en décembre 2010. Roger Bastide, "Approche interdisciplinaire de la maladie mentale ", Social Science Information, vol. 6, $\mathrm{n}^{\circ} 4,1967$, p. 37-52.

21 Heinz Heckhausen, "Discipline et interdisciplinarité ", dans OCDE, L'interdisciplinarité, problème d'enseignement et de recherche, 1972, p. 83-90.

22 Marcel H. Boisot, "Discipline et interdisciplinarité ", dans OCDE, L'interdisciplinarité, problème d'enseignement et de recherche, 1972, p. 90-97.

Erich Jantsch, «Vers l'interdisciplinarité et la transdisciplinarité dans l'enseignement et l'innovation ", dans OCDE, L'interdisciplinarité, problème d'enseignement et de recherche, 1972, p. 98-125. 
multidisciplinarité ou pluridisciplinarité), le deuxième, une structure aux trois paliers que sont l'interdisciplinarité téléologique, l'interdisciplinarité normative et l'interdisciplinarité objectivisée, et le troisième, la transdisciplinarité;

iii. celle de Jean Piaget $^{24}$, qui présente trois niveaux : le multidisciplinaire, l'interdisciplinaire et le transdisciplinaire;

iv. celle de Guy Palmade ${ }^{25}$, où sont énumérées pas moins de huit variétés : multidisciplinaire et pluridisciplinaire, interdisciplinarité auxiliaire, interdisciplinarité d'intersection et d'interdépendance, interdisciplinarité d'emboîtement, transdisciplinarité locale, transdisciplinarité étendue, transdisciplinarité générale, et co-disciplinarité.

Creutzer Mathurin fait aussi état des propos de Gilbert Durand $^{26}$, Yves Bertrand ${ }^{27}$, Yvon Gauthier ${ }^{28}$, Earl J. McGrath ${ }^{29}$, Jacques Brazeau ${ }^{30}$, Jean-Paul Resweber ${ }^{31}$, Jean-Marie Benoist ${ }^{32}$,

24 Jean Piaget, "L'épistémologie des relations interdisciplinaires », dans OCDE, L'interdisciplinarité, problème d'enseignement et de recherche, 1972, p. 131144.

25 Guy Palmade, Interdisciplinarité et idéologies, Paris, Anthropos, 1977.

26 Gilbert Durand, "Multidisciplinarité et heuristique ", dans OCDE, L'interdisciplinarité, problème d'enseignement et de recherche, 1972, p. 25-26.

27 Yves Bertrand, "L'interdisciplinarité ", Pédagogiques, vol. 1, n 1, 1976, p. 3-5; "Le problème, c’est la disciplinarité ", Pédagogiques, vol. 1, n 4, 1976, p. 10-11.

28 Yvon Gauthier, "La logique et l'épistémologie des sciences à la charnière multidisciplinaire ", Pédagogiques, vol. 1, n 4, 1976, p. 6-9.

29 Earl J. McGrath, "Interdisciplinary Studies: An Integration of Knowledge and Experience ", Change. The Magazine of Learning, vol. 10, $\mathrm{n}^{\circ}$ 6, 1978, p. 6-9.

30 Jacques Brazeau, "L'interdisciplinarité et les études supérieures ", Sociologie et sociétés, vol. 12, $\mathrm{n}^{\circ} 2,1980$, p. 97-106.

31 Jean-Paul Resweber, La méthode interdisciplinaire, Paris, PUF, 1981.

32 Jean-Marie Benoist, "L'interdisciplinarité dans les sciences sociales ", dans Interdisciplinarité et sciences humaines, Paris, UNESCO, 1983, p. 169-190. 
Stanislav N. Smirvov ${ }^{33}$, Georges Gusdorf ${ }^{34}$, Félix Guattari ${ }^{35}$ et Margaret A. Somerville ${ }^{36}$. Tous ces auteurs discutent de taxinomies, critiquant celles qui existent déjà ou suggérant d'autres classifications. Creutzer Mathurin fait remarquer, entre autres choses, que ces typologies ont un vocabulaire relativement commun et qu'elles tendent à être hiérarchiques.

Dans des études plus récentes sur l'interdisciplinarité, on trouve encore des variations sur le thème de la typologie. Julie Thompson Klein vient de publier un texte dans lequel elle discute du vocabulaire qui est corrélé aux termes dominants des typologies (multidisciplinarité, interdisciplinarité et transdisciplinarité) ${ }^{37}$. Wolfgang Khron vient de distinguer trois formes d'interdisciplinarité : interdisciplinary case work, interdisciplinary communication et interdisciplinary fusion ${ }^{38}$. Steve Fuller vient de présenter six formes d'interdisciplinarité déviante ${ }^{39}$.

33 Stanislav N. Smirnov, "L'approche interdisciplinaire dans la science d'aujourd'hui : fondements ontologiques et épistémologiques, formes et fonctions ", dans Interdisciplinarité et sciences humaines, Paris, UNESCO, 1983, p. 53-71.

34 Georges Gusdorf, "Réflexion sur l'interdisciplinarité », Bulletin de psychologie, tome 43, n 397, 1990, p. 869-895.

35 Félix Guattari, "Fondements éthico-politiques de l'interdisciplinarité », dans Edouardo Portella (dir.), Entre savoirs. L'interdisciplinarité en acte : enjeux, obstacles, résultats, Toulouse, Érès / Paris, UNESCO, 1992, p. 101-109. Margaret A. Somerville, «La transdisciplinarité, vague de l'avenir : comment préparer nos rivages à l'accueillir ", dans Edouardo Portella (dir.), Entre savoirs. L'interdisciplinarité en acte : enjeux, obstacles, résultats, Toulouse, Érès / Paris, UNESCO, 1992, p. 117-136.

37 Julie Thompson Klein, "A Taxonomy of Interdisciplinarity ", dans Robert Frodeman, Julie Thompson Klein et Carl Mitcham (dir.), The Oxford Handbook of Interdisciplinarity, Oxford, Oxford University Press, 2010, p. 15-30.

38 Wolfgang Krohn, "Interdisciplinary Cases and Disciplinary Knowledge ", dans Robert Frodeman, Julie Thompson Klein et Carl Mitcham (dir.), The Oxford Handbook of Interdisciplinarity, Oxford, Oxford University Press, 2010, p. 31-41.

39 Steve Fuller, "Deviant Interdisciplinarity ", dans Robert Frodeman, Julie Thompson Klein et Carl Mitcham (dir.), The Oxford Handbook of Interdisciplinarity, Oxford, Oxford University Press, 2010, p. 50-64. 


\section{Une typologie n'est pas une théorie}

La question qui se pose au début de cet article ne concerne pas la théorisation interdisciplinaire; la question qui se pose est celle de l'existence d'une théorie sur l'interdisciplinarité. Or, il n'y a pas à se demander s'il y a des théories interdisciplinaires. Il y en a de nombreuses. Les sciences humaines réalisent des œuvres interdisciplinaires en grand nombre au plan empirique aussi bien que théorique. Elles sont amenées à le faire bien simplement parce qu'une infinité d'interrogations ne peuvent trouver de réponse dans le cadre d'une seule discipline. Cette multitude de produits scientifiques n'est pas indépendante de l'intérêt que de nombreux penseurs accordent au fait de l'interdisciplinarité. Nous avons vu que cet intérêt générait un discours qui prenait trois directions.

Nous avons noté qu'il constituait une réaction contre la spécialisation, un projet de dépassement des limites de la logique cartésienne, d'intégration des connaissances. Nous avons remarqué que ce projet pouvait se traduire en un mouvement aux fins praxéologiques et éthiques, voire utopiques.

Nous avons constaté que l'interdisciplinarité était souvent présentée dans des logiques binaires et qu'elle était alors simplement l'autre de quelque chose.

Nous avons enfin observé qu'une large part des travaux sur l'interdisciplinarité ne constituaient, en fait, que des typologies.

Il importerait sans doute, dans un autre contexte, de revenir sur toutes ces réflexions pour rappeler, par exemple :

i. que l'intégration n'est pas le monopole de l'interdisciplinarité;

ii. que les logiques systémiques peuvent servir des recherches monodisciplinaires;

iii. que les constats du mouvement transdisciplinaire sur l'état du monde sont trop peu nuancés;

iv. qu'on peut séparer et réunir aussi bien en interdisciplinarité qu'en monodisciplinarité; 
v. que l'analyse qualitative et l'analyse quantitative appartiennent et à la monodisciplinarité et à l'interdisciplinarité;

vi. qu'il ne peut pas y avoir de science en dehors de la désubjectivisation.

Mais cela nous éloignerait de la question à laquelle nous devons répondre : y a-t-il une théorie de l'interdisciplinarité en sciences humaines?

Démontrer que l'interdisciplinarité est nécessaire dans le champ scientifique, opposer l'interdisciplinarité à la monodisciplinarité, classer les formes d'interdisciplinarité, ce n'est pas faire une théorie de l'interdisciplinarité. Une théorie, c'est un agencement logique de concepts qui permet d'expliquer quelque chose. C'est bien ce que font les travaux interdisciplinaires. Ce n'est pas ce que font les travaux sur l'interdisciplinarité. Plaider, ce n'est pas théoriser. Classer, ce n'est pas théoriser. Ce n'est pas pour rien que l'essentiel du travail sur l'interdisciplinarité, ce sont des typologies; c'est qu'on peut catégoriser ce qu'on observe dans les travaux interdisciplinaires, mais qu'on ne peut pas théoriser cet objet. Que pourrait d'ailleurs être une théorie sur l'interdisciplinarité? L'objet est à ce point divisible et pluriel que les récurrences ne peuvent être que sectorielles. À quoi même pourrait servir une telle théorie? Pourrait-il s'agir d'une épistémologie? Une telle épistémologie montrerait que la vérité - au sens de qualité qu'on attribue à une proposition ou à un ensemble d'énoncés - en interdisciplinarité est différente de ce qu'elle est en monodisciplinarité. Or, on constate rapidement que les résultats des recherches interdisciplinaires se soumettent aux mêmes impératifs que ceux des analyses monodisciplinaires : consistance, exhaustivité, limitation des objets, herméneutique ou prédiction, vérification ou falsification, logique interne, irréductibilitét ${ }^{40}$. C'est pour ces raisons, nous semble-t-il, que les travaux sur l'interdisciplinarité ne sont pas à la hauteur des travaux interdisciplinaires.

$\overline{40}$ Sur ces critères, voir Yvon Gauthier, Entre science et culture. Introduction à la philosophie des sciences, Montréal, Presses de l'Université de Montréal, coll. "Paramètres ", 2005. 
Il ne peut pas plus y avoir une théorie de l'interdisciplinarité qu'une théorie de la science. L'épistémologie peut aider à comprendre comment les scientifiques produisent leurs travaux; elle peut établir des critères de vérité; elle n’est pas théorie de la science; et ce qu'elle découvre vaut pour la recherche autant monodisciplinaire qu'interdisciplinaire.

\section{Conclusion}

Il y a bien des épistémologies, des tentatives de départager le scientifique du non-scientifique, mais il n'y a pas de science de la science, de théorie de la science, de théorie de la monodisciplinarité ou de théorie de l'interdisciplinarité. La science se réalise dans la pluralité sociohistorique des rapports entre des scientifiques, des discours scientifiques et des objets de science. Ces dialectiques sont monodisciplinaires autant qu'interdisciplinaires. Et c'est sans doute parce qu'elle n'est pas théorisable sur ce plan qu'elle est ce qu'elle est. Il importe que la science comprenne ce qui se donne à elle, dans des dialectiques particulières. Il importe que la science réfléchisse sur elle-même. Mais importe-t-il que tout le savoir scientifique se réduise à une théorie? À quoi même pourrait servir cette théorie? Penser de tels ensembles, c'est normalement les classer; et il peut difficilement en être autrement. C'est pourquoi l'épistémologie se donne souvent pour mission de distinguer le scientifique du non-scientifique.

L'interdisciplinarité produit des théories, comme le fait la monodisciplinarité. Il ne peut pas y avoir une théorie de ces théories, à moins qu'elle soit désincarnée, vidée de sa substance, qu'elle soit à ce point formelle qu'elle n'ait plus de thématique, qu'elle élimine la triple dialectique des scientifiques, des discours scientifiques et des objets de science. Il importe à la science de réfléchir sur la science, sur les théories qui sont produites sociohistoriquement dans le champ scientifique, qu'il soit monodisciplinaire ou interdisciplinaire. Mais une théorie du champ interdisciplinaire serait probablement aussi utile à l'interdisciplinarité - et à l'humain - qu'une métaphysique à la physique. 


\section{Bibliographie}

Bastide, Roger, "Approche interdisciplinaire de la maladie mentale », Social Science Information, vol. 6, n 4, 1967, p. 37-52.

Benoist, Jean-Marie, "L'interdisciplinarité dans les sciences sociales », dans Interdisciplinarité et sciences humaines, Paris, UNESCO, 1983, p. 169190.

Bertrand, Yves, "L'interdisciplinarité ", Pédagogiques, vol. 1, n 1, 1976, p. 3-5.

Bertrand, Yves, "Le problème, c'est la disciplinarité ", Pédagogiques, vol. $1, \mathrm{n}^{\circ} 4,1976$, p. $10-11$.

Boisot, Marcel H., "Discipline et interdisciplinarité ", dans OCDE, L'interdisciplinarité, problème d'enseignement et de recherche, 1972, p. 90-97.

Brazeau, Jacques, «L'interdisciplinarité et les études supérieures ", Sociologie et sociétés, vol. 12, nº 2, 1980, p. 97-106.

Camus, Michel, "Au-delà des deux cultures : la voie transdisciplinaire ", dans Groupe 21, Mémoire du XXIe siècle, Monaco, Éditions du Rocher, 1999, p. 25-34.

Charte de la transdisciplinarité, http://basarab.nicolescu.perso.sfr.fr/ciret/ chartfr.htm, 1994, Premier congrès mondial de la transdisciplinarité, site consulté en décembre 2010.

Durand, Gilbert, "Multidisciplinarité et heuristique ", dans OCDE, L'interdisciplinarité, problème d'enseignement et de recherche, 1972, p. 25-26.

Finkenthal, Michael, Interdisciplinarity: Toward the Definition of a Metadiscipline?, New York, Peter Lang, coll. "American University Studies", Series V, Philosophy, vol. 187, 2001.

Fuller, Steve, "Deviant Interdisciplinarity ", dans Robert Frodeman, Julie Thompson Klein et Carl Mitcham (dir.), The Oxford Handbook of Interdisciplinarity, Oxford, Oxford University Press, 2010, p. 50-64.

Gauthier, Yvon, Entre science et culture. Introduction à la philosophie des sciences, Montréal, Presses de l'Université de Montréal, coll. « Paramètres ", 2005.

Gauthier, Yvon, « La logique et l'épistémologie des sciences à la charnière multidisciplinaire ", Pédagogiques, vol. 1, n 4, 1976, p. 6-9.

Guattari, Félix, "Fondements éthico-politiques de l'interdisciplinarité ", dans Edouardo Portella (dir.), Entre savoirs. L'interdisciplinarité en acte: enjeux, obstacles, résultats, Toulouse, Érès / Paris, UNESCO, 1992, p. 101-109. 
Gusdorf, Georges, «Réflexion sur l'interdisciplinarité », Bulletin de psychologie, tome 43, $\mathrm{n}^{\circ} 397,1990$, p. 869-895.

Heckhausen, Heinz, "Discipline et interdisciplinarité ", dans OCDE, L'interdisciplinarité, problème d'enseignement et de recherche, 1972, p. 83-90.

Jantsch, Erich, "Vers l'interdisciplinarité et la transdisciplinarité dans l'enseignement et l'innovation ", dans OCDE, Linterdisciplinarité, problème d'enseignement et de recherche, 1972, p. 98-125.

Krohn, Wolfgang, "Interdisciplinary Cases and Disciplinary Knowledge ", dans Robert Frodeman, Julie Thompson Klein et Carl Mitcham (dir.), The Oxford Handbook of Interdisciplinarity, Oxford, Oxford University Press, 2010, p. 31-41.

Mathurin, Creutzer, «Aspects de l'interdisciplinarité : essai de reconstitution d'un débat ", dans Lucie Gélineau et Carole Mailloux (dir.), Linterdisciplinarité et la recherche sociale appliqué. Réflexions sur des expériences en cours, Université de Montréal, Université Laval et Chaire d'étude Claire-Bonenfant sur la condition des femmes, octobre 2002, p. 7-39, http://www.fas.umontreal.ca/sha/documents/interdisciplinarite.pdf, site consulté en décembre 2010.

McGrath, Earl J., «Interdisciplinary Studies: An Integration of Knowledge and Experience ", Change. The Magazine of Learning, vol. 10, $\mathrm{n}^{\circ} \mathrm{6}$, 1978, p. 6-9.

Morin, Edgar, "L'épistémologie de la complexité ", dans Edgar Morin et Jean-Louis Le Moigne, Lintelligence de la complexité, Paris, L'Harmattan, coll. " Cognition et formation ", 1999, p. 43-169.

Morin, Edgar, La méthode, tomes 1 à 4, Paris, Seuil, 1977-1991.

Morin, Edgar, La méthode, tome 5, L'humanité de l'humanité, Paris, Seuil, 2001.

Morin, Edgar, La méthode, tome 6, L'éthique, Paris, Seuil, 2006.

Morin, Edgar, «La pensée complexe, une pensée qui se pense ", dans Edgar Morin et Jean-Louis Le Moigne, Lintelligence de la complexité, Paris, L'Harmattan, coll. "Cognition et formation ", 1999, p. 247-267.

Morin, Edgar, Science et conscience, Paris, Seuil, coll. " Points Essais", 1990.

Morin, Edgar, La tête bien faite, Paris, Seuil, 1999.

Nicolescu, Basarab, La Transdisciplinarité. Manifeste, Monaco, Éditions du Rocher, coll. "Transdisciplinarité », 1996.

Nietzsche, Friedrich, "Nous, les savants ", dans Par-delà le bien et le mal, Paris, Union générale d'éditions, coll. «10/18 », [1725] 1970, p. 133152. 
Nissani, Moti, «Fruits, Salads and Smoothies: A Working Definition of Interdisciplinarity ", Journal of Education Thought, vol. 29, n 2, août 1995, p. 121-128.

Ortega y Gasset, José, "La barbarie du "spécialisme” ", dans La révolte des masses, Paris, Gallimard, coll. "Idées », [1930] 1961, p. 158-159.

Palmade, Guy, Interdisciplinarité et idéologies, Paris, Anthropos, 1977.

Piaget, Jean, "L'épistémologie des relations interdisciplinaires ", dans OCDE, Linterdisciplinarité, problème d'enseignement et de recherche, 1972, p. 131-144.

Resweber, Jean-Paul, La méthode interdisciplinaire, Paris, PUF, 1981.

Smirnov, Stanislav N., "L'approche interdisciplinaire dans la science d'aujourd'hui : fondements ontologiques et épistémologiques, formes et fonctions ", dans Interdisciplinarité et sciences humaines, Paris, UNESCO, 1983, p. 53-71.

Somerville, Margaret A., "La transdisciplinarité, vague de l'avenir : comment préparer nos rivages à l'accueillir ", dans Edouardo Portella (dir.), Entre savoirs. L'interdisciplinarité en acte : enjeux, obstacles, résultats, Toulouse, Érès / Paris, UNESCO, 1992, p. 117-136.

Thompson Klein, Julie, Interdisciplinarity: History, Theory, and Practice, Détroit, Wayne State University, 1990.

Thompson Klein, Julie, "A Taxonomy of Interdisciplinarity ", dans Robert Frodeman, Julie Thompson Klein et Carl Mitcham (dir.), The Oxford Handbook of Interdisciplinarity, Oxford, Oxford University Press, 2010, p. 15-30.

Vico, Giambattista, La science nouvelle, Paris, Gallimard, coll. "Tel ", [1725] 1993.

Walker Vinyard, Alma, «Identification and Self-Definition: A Twenty-First Century Agenda for Interdisciplinary and International Women's Studies ", dans Kate Conway-Turner, Suzanne Cherrin, Jessica Schiffman, and Kathleen Doherty Turkel (dir.), Women's Studies in Transition. The Pursuit of Interdisciplinarity, Newark, University of Delaware Press / London, Associated University Press, 1998, p. 92-101.

Weingart, Peter, "Interdisciplinarity: The Paradoxical Discourse ", dans Peter Weingart et Nico Stehr (dir.), Practising Interdisciplinarity, Toronto, Buffalo, London, University of Toronto Press, 2000, p. 25-41. 